\title{
An Inter-vehicle Communication Based Cooperative Speed Suggestion Algorithm for Smoothing Fluctuations in Stop-and-go Traffic
}

\author{
Dong Wang, Jiancong Sun, Xiaohong Li, Zhu Xiao \\ College of Information Science and Engineering \\ Hunan University \\ Changsha, China \\ sjc_329@163.com
}

\begin{abstract}
Stop-and-go traffic can cause a significant amount of pollutant emissions due to frequent accelerations and decelerations. Various efforts have been made to smooth traffic flow and reduce air emissions and fuel consumption in stopand-go conditions. In this paper, we propose a cooperative algorithm based on inter-vehicle communications (IVCs) to suggest advisory speed for the purpose of smoothing vehicle's speed profile without sacrificing the transportation efficiency. By utilizing the preceding vehicle's information, the algorithm extract the period of fluctuations and estimate the average speed of traffic flow to provide an appropriate speed suggestion. In simulation, our algorithm is evaluated with realworld traffic data. The results demonstrate the effectiveness of our algorithm and show that the $\mathrm{CO}_{2}$ emissions can be reduced by up to $26.9 \%$ while the fuel consumption can be saved by $24 \%$ to $28 \%$.
\end{abstract}

Keywords: Inter-vehicle Communication, Advisory Speed, Stop-and-go Traffic

\section{INTRODUCTION}

The transportation sector in the United States accounts for one third of greenhouse gas emissions, of which $80 \%$ is from cars and trucks in roadway systems [1]. A significant amount of pollutant emissions and fuel consumption are made due to traffic oscillations including high acceleration, congestion and stop-and-go conditions [2]. It has been found that by reducing traffic oscillations, especially eliminate frequent acceleration and deceleration events associated with stop-and-go traffic that exists during congested conditions, $\mathrm{CO}_{2}$ emissions can be reduced by up to $20 \%$ [3]

Many efforts are made to reduce traffic oscillations, such as changeable speed signs, eco-driving, and ISA system. The changeable speed signs are devices used to display variable speed limits for traffic flow readjustment. The variable speed limits are calculated based on the average speed of traffic [4], the shockwave prediction algorithms and environment factors [5], the traffic flow rates and congestion levels [6] or even the model predictive control approach [7]. It is shown that these methods significantly improve the stability of traffic by forcing drivers to obey the speed restriction. Instead of mandatory policies, eco-driving attempt to change drivers' behaviors through general advice, such as: do not drive too fast, do not accelerate too quickly, maintain steady speed, and keep vehicle in good maintenance. It is worth noting that nearly all eco-driving-related research to date has been on providing static advice to drivers and measuring before-and-after differences [8]. With the development of wireless communication, suggesting or enforcing speed limits for individual vehicle becomes possible. The Intelligent Speed Adaptation (ISA) system [9] is an example of providing advisory speed for individuals to smooth vehicle's trajectory in congested traffic. It can potentially mitigate traffic congestion by leveraging road congestion information.

Inter-vehicle communication (IVC) technologies enable vehicles to exchange information through dedicated shortrange communications (DSRC) devices. Sensors and antennas are equipped by cars and trucks which participate in VANETs (Vehicular Ad Hoc Networks). These devices can be used to develop advanced information systems [10] in cooperative approach. Recently, a control theoretic formulation within the framework of feedback control systems has been proposed to smooth traffic flow [11].

In this paper, we propose an IVC-based cooperative algorithm and evaluate it with real-world traffic data. This algorithm is designed to provide a real-time advisory speed for a manually driven vehicle to smooth the movements and reduce air pollution and fuel consumption in stop-and-go fluctuations without sacrificing the transportation efficiency. In our study, Newell's car-following model [12] is used to limit vehicle speed for safety, and Fast Fourier transform (FFT) algorithm is used to obtain the frequency-domain characteristics. We also evaluate the smoothing effect of our algorithm using the Virginia Tech microscopic energy and emission model (VT-Micro) [13].

The rest of this paper is organized as follows. Section II states the problems, assumptions and goals that our algorithm is designed for. In Section III, we introduce Newell's car-following model, FFT and VT-Micro as modules and preparation knowledge in our algorithm. We then propose the cooperative speed suggestion algorithm in Section IV and evaluate it with real trajectory data in Section V. Section VI concludes this paper and gives directions for future work.

\section{PROBLEM STATEMENT}

\section{A. Problems}

On an open single-lane road, a vehicle platoon starts with vehicle number 1 is following a leading vehicle 0 . The leading vehicle, somehow, is traveling on stop-and-go traffic waves with frequent accelerations and decelerations. One or more vehicles in the vehicle platoon including vehicle 1 are 
equipped with IVC devices. A cooperative speed suggestion algorithm should be designed to smooth the trajectories and reduce air pollution and fuel consumption of these vehicles.

\section{B. Assumptions}

(1) We assume that the periods of oscillations are no more than 4 minutes since our experiment results show the smoothing effect is not significant and has side effect when the periods exceed 4 minutes because the accelerations and decelerations are infrequent. Some studies also show that traffic oscillations in stop-and-go traffic are normally periodic with a period less than 4 minutes [14][15].

(2) We make the assumption that every following vehicle in this scenario is capable of detecting or receiving status information including position and velocity from its preceding vehicle.

C. Goals

(1) Vehicle should travel smoother after applying this algorithm. That is, vehicle travels with smaller speed variation, less air emissions and fuel consumption.

(2) The vehicle's average speed should not decrease. Otherwise the traffic flow may be slowed down, and the transportation efficiency will be sacrificed.

(3) The algorithm should take effect even if only one vehicle is following its leader.

(4) A vehicle that implement this algorithm should not be interfered by other vehicles which are following behind since these followers' behavior is highly unpredictable.

(5) A vehicle should benefit from the information that is shared from those vehicles in front of it and get better effect of smoothing.

\section{ALGORITHM MODULE DESCRIPTION}

In this section, we introduce Newell's car-following model for maintaining vehicle's speed limitation and FFT for period extraction. In addition, VT-Micro will be introduced to evaluate the environment impact after smoothing.

\section{A. Newell's car-following model}

Newell's car-following model is a simplified numerical solution to describe vehicle's movements. According to previous literature, this model is considered to be able to perform as well as complex models [16], and has been verified in real-world traffic under several circumstances [17]. In our study, we use Newell's model to derive the realtime maximum safe speed of vehicles.

On a one-lane road, if vehicle $n$ is following vehicle $n-1$, the trajectory of vehicle $n$ can be denoted as [12][18]

$$
x_{n}\left(t+\tau_{n}\right)=\min \left\{x_{n-1}(t)-d_{n}, x_{n}(t)+v_{f} \tau_{n}\right\} \text { (1) }
$$

where $d_{n}$ is the jam spacing, $v_{f}$ is the free-flow speed, and $\tau_{n}$ is the response delay. Equation (1) implies that a following vehicle will manage to advance its position as far as possible, while keeping a distance no less than $d_{n}$ from the leading vehicle without exceeding the speed limit $v_{f}$. That is, in congestion a vehicle will translate the leader's trajectory in space and time by a displacement $d_{n}$ and $\tau_{n}$, while in uncongested traffic vehicle will travel at free-flow speed.

When vehicles move at a constant speed, the equation $v_{n}\left(t+\tau_{n}\right)=\frac{x_{n}\left(t+\tau_{n}\right)-x_{n}(t)}{\tau_{n}}$ is satisfied, then Newell's model can be written as

$$
v_{n}\left(t+\tau_{n}\right)=\min \left\{\frac{x_{n-1}(t)-x_{n}(t)-d_{n}}{\tau_{n}}, v_{f}\right\}
$$

where the first regime of this equation provides a carfollowing condition which can be used to determine if a vehicle is following its leader. When vehicle's trajectory is smoothed, $v_{n}\left(t+\tau_{n}\right)=\frac{x_{n-1}(t)-x_{n}(t)-d_{n}}{\tau_{n}}$ will not be satisfied. Anyhow, (2) can be used to calculate the real-time speed limit for safety.

Since every vehicle in this paper is identical, we use $d$ and $\tau$ short for $d_{n}$ and $\tau_{n}$.

\section{B. Fast Fourier transform (FFT)}

Fast Fourier transform is an algorithm to convert time (or space) to frequency and its inverse rapidly [19]. In our study, FFT is used to transform time-domain speed profiles into frequency-domain signals for analysis.

We utilize FFT algorithm as follow. The input is an array of velocity data $v[N]$ in sample rate $f_{s}$ and size $N$. The output of FFT, $X[N]=\operatorname{FFT}(v[N])$, will be an N-length array of complex numbers. By computing the modulus of each number in $X[N]$ and dividing them by $N / 2$ (or $N$, if and only if it is the modulus of the first element in $X[N]$ ), we will get the result as an array $A[N]$ consists of $N$ real numbers. The first number of $A[N]$, that is, $A_{0}$, is the average speed in the scope of $N$. And every $A_{k} \in A[N],(k=1, \ldots, N / 2-1)$ represents the amplitude on frequency $k \times f_{s} / N$. By this means, we can find the largest $A_{k}$ with $k \in[1, N / 2-1]$ and consider the frequency $k \times f_{s} / N$ as the main frequency.

Although we can extract the period of fluctuations by calculating reciprocal of the main frequency, it is worth noting that the resolution of the spectrum is not high enough, especially between $f_{s} / N$ and $2 \times f_{s} / N$. Another refining process should take place.

\section{The Virginia Tech microscopic energy and emission model (VT-Micro)}

Numerous fuel consumption and emission models have been developed over the past decade. State-of-the-art emission models are categorized as either macroscopic or microscopic. Macroscopic models, such as MOBILE and EMFAC, are capable of estimating network-wide emission 
rates. Alternatively, microscopic models, such as CMEM and VT-Micro, can estimate instantaneous vehicle pollutant emissions and fuel consumption second-by-second. In our study, we use VT-Micro model for evaluation since VTMicro takes speeds and acceleration rates as input which can be simply incorporated.

VT-Micro is developed as a regression model from experimentation with numerous polynomial combinations of speed and acceleration levels. It is demonstrated by [13]

$$
\ln M O E_{\mathrm{m}}= \begin{cases}\sum_{i=0}^{3} \sum_{j=0}^{3} M_{i j}^{\mathrm{m}} \times v^{i} \times a^{j}, & a \geq 0 \\ \sum_{i=0}^{3} \sum_{j=0}^{3} L_{i j}^{\mathrm{m}} \times v^{i} \times a^{j}, & a<0\end{cases}
$$

where

$M O E_{\mathrm{m}}=$ Instantaneous fuel consumption or emission rate,

$M_{i j}^{\mathrm{m}}=$ Model regression coefficient for $M O E_{\mathrm{m}}$ at speed power $i$ and acceleration power $j$ for positive accelerations, $L_{i j}^{\mathrm{m}}=$ Model regression coefficient for $M O E_{\mathrm{m}}$ at speed power $i$ and acceleration power $j$ for negative accelerations,

$v=$ Instantaneous speed,

$a=$ Instantaneous acceleration rate.

In the evaluation, we calculate the speeds and acceleration rates of every vehicle in simulation and compute air emissions and fuel consumption with the VT-Micro software [20] released by Dr. Hesham Rakha. We also take standard deviation as a key criterion into consideration. By comparing these profiles, we can demonstrate the effectiveness of our algorithm.

\section{PROPOSED ALGORITHM}

In this section, we propose a cooperative speed suggestion algorithm which calculate the advisory speed in seven steps. The block diagram of this algorithm is shown in Figure 1. The algorithm is developed for both continuous time and discrete time, but for simplicity, we consider the time as discrete and the time interval is 1 second.

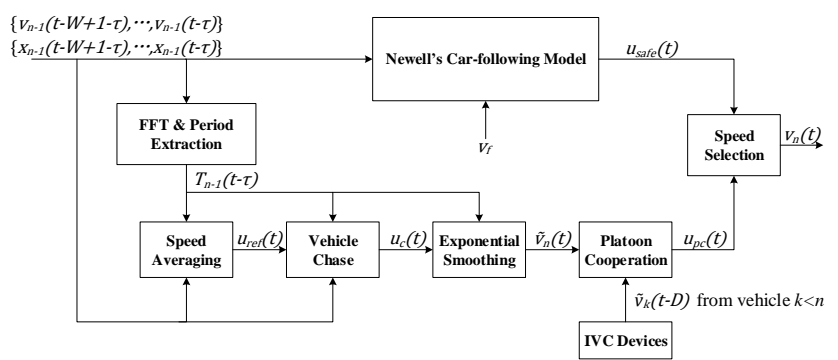

Figure 1. The block diagram of the algorithm

\section{1) Safe speed calculation}

In this step, the real-time maximum safe speed is calculated by using Newell's car-following model. As discussed in Section III, we have

$$
u_{\text {safe }}(t)=\min \left\{\frac{x_{n-1}(t-\tau)-x_{n}(t-\tau)-d}{\tau}, v_{f}\right\}
$$

where $u_{\text {safe }}(t)$ is the safe speed limit of vehicle $n$ at time $t$.

2) Period extraction

To acquire the precise average speed that the preceding vehicle $n-1$ is traveling at during a stop-and-go period, we need to extract the period of fluctuations, $T_{n-1}(t-\tau)$, of vehicle $n-1$. As we assumed in Section II, periods without exceed $60 \times 4$ seconds are in concern. Thus, we set up a Wlength window for sampling velocity data from vehicle $n-1$ and $\mathrm{W}$ is equal to 256. As detailed in Section III, when $t \geq W+\tau-1$, with $f_{s}=1 H z$ and $N=W$, a main frequency point can be selected by using FFT but still in need of refining.

With different $k \in[1, N / 2-1]$ that makes $A_{k}$ the largest number in $A[N]$, we determine a possible period range

$$
\left[P_{L}, P_{H}\right]=\left\{\begin{array}{lr}
{[\lceil\lceil/ 1.5\rceil, 240],} & k=1 \\
{[\lceil W /(k+0.5)],[W /(k-0.5)]],} & 2 \leq k \leq 7 \\
\lceil\lceil W / k\rceil,\lceil W / k\rceil], & 8 \leq k
\end{array}\right.
$$

where $P_{L}$ and $P_{H}$ are the boundaries of the range. Since the sum values in a precise period within the $\mathrm{W}$-length window should be approximately equal, a function can be set as

$$
f(p)=\left|\sum_{k=t-W+1}^{t-W+p} v_{n-1}(k-\tau)-\sum_{k=t-p+1}^{t} v_{n-1}(k-\tau)\right|
$$

It is a simple way to estimate the period. $\forall P \in\left[P_{L}, P_{H}\right], \exists P_{0}$ that can satisfy $f\left(P_{0}\right) \leq f(P)$ is the period we expect. If multiple $P_{0}$ exist, the largest one should be chosen. Additionally, when $t<W+\tau-1$, there is no enough information for period extraction, the period will be considered as $\lceil(t-\tau+1) / 2\rceil$ to track the latest movements of the preceding vehicle. Now we can ultimately determine the period of vehicle $n-1$ at time $t-\tau$

$$
T_{n-1}(t-\tau)=\left\{\begin{array}{cc}
[t-\tau+1) / 2\rceil, & t<W+\tau-1 \\
P_{0}, & \text { otherwise }
\end{array}\right.
$$

3) Speed averaging

With the period calculated in the previous step, we can obtain the average speed of the preceding vehicle as our referential speed by

$$
u_{\text {ref }}(t)=\frac{1}{T_{n-1}(t-\tau)} \sum_{k=t-T_{n-1}(t-\tau)+1}^{t} v_{n-1}(k-\tau)
$$

4) Vehicle chase

If vehicle $n$ is failed to follow vehicle $n-1$, transportation efficiency will deteriorate. Thus, we dynamically increase the referential speed by

$\Delta u_{c}(t)=\left\{\begin{array}{cc}0, & t<W+\tau \\ \frac{1}{T_{n-1}(t-\tau)} \times \min _{k=t-T_{n-1}(t-\tau)}\left\{\frac{x_{n-1}(k-\tau)-x_{n}(k-\tau)-d}{\tau}-v_{n}(k)\right\}, & \text { otherwise }\end{array}\right.$ 
According to the discussion in Section III, after time $W+\tau, \Delta u_{c}(t)=0$ only if vehicle $n$ follows vehicle $n-1$ at least once during a stop-and-go period, otherwise $\Delta u_{c}(t)>0$. Therefore, we adjust vehicle's speed to catch up with its leader in a period. By now, we can set the speed of chase as

$$
u_{c}(t)=u_{r e f}(t)+\Delta u_{c}(t)
$$

\section{5) Exponential smoothing}

In this step, we take the exponential moving averaging of $u_{c}$ to minimize the variation of speed. We choose $\alpha e^{-\alpha i}$, $i \in[0,+\infty)$ as the weighting factor since $\int_{0}^{+\infty} \alpha e^{-\alpha x} d x=1$ and $f(x)=\alpha e^{-\alpha x}$ is a decreasing function that gives more weight to the most recent $u_{c}$. In order to control the weight distribution, we define $w$ as the cumulative weight of $\alpha e^{-\alpha x}$ in $\left(0, T_{0}\right)$ which is the most recent period, and we have $\alpha=\ln (1-w) /\left(-T_{0}\right)$, where $T_{0}$ in this step is equal to $T_{n-1}(t-\tau)$. A larger $w$ would cause the advisory speed to be more responsive to recent changes of $u_{c}$. In our study, $w$ is empirically decided to be a constant value 0.75 . The smoothed speed $\tilde{v}_{n}(t)$ is calculated by

$$
\tilde{v}_{n}(t)=\left\{\begin{array}{cc}
u_{c}(t), & t<W / 2+\tau-1 \\
\frac{\sum_{k=W / 2+\tau-1}^{t} \alpha e^{-\alpha(t-k)} u_{c}(k)}{\sum_{k=0}^{t-W / 2-\tau+1} \alpha e^{-\alpha k}}, & \text { otherwise }
\end{array}\right.
$$

\section{6) Platoon cooperation}

In this step, vehicles share/receive $\tilde{v}$ with/from each other. As mentioned in Section II, a vehicle should only care about those vehicles in front of it. Thus, by combining vehicles' $\tilde{v}$, we average the speeds in the platoon and compute the cooperative speed by

$$
u_{p c}(t)=\frac{\tilde{v}_{n}(t)+\sum_{k<n} \tilde{v}_{k}(t-D)}{K+1}
$$

where $\tilde{v}_{k}$ represents the $\tilde{v}$ of vehicle $k$ which is in front of vehicle $n$ and equipped with IVC devices, $D$ is the communication delay of exchanging and integrating data, and $K$ is the total number of vehicles which are in front of vehicle $n$ and sharing informations.

\section{7) Speed Selection}

The last step is the speed selection. It is necessary because the cooperative speed $u_{p c}(t)$ should not exceed the safe limit $u_{\text {safe }}(t)$. The output of our algorithm, the final advisory speed $v_{n}(t)$ is

$$
v_{n}(t)=\min \left\{u_{p c}(t), u_{\text {safe }}(t)\right\}
$$

\section{Simulation}

In this section, we evaluate our algorithm by measuring several assessment criteria of velocity, emissions and consumption. A simulation platform has been established with a single-lane road scenario. In this simulation, four vehicles including a leading vehicle (vehicle 0 ) and three followers (vehicle 1, 2, 3) are traveling on the road for 1200 seconds. The trajectory of the leading vehicle is provided, and the parameter settings of this simulation are shown in Table 1.

Table 1. Parameter settings of simulation

\begin{tabular}{|c|c|}
\hline Parameters & Values \\
\hline$v_{f}$, Free-flow speed & $30(\mathrm{~m} / \mathrm{s})$ \\
\hline$d$, Jam spacing & $7.25(\mathrm{~m})$ \\
\hline$\tau$, Time displacement & $1(\mathrm{~s})$ \\
\hline$D$, Communication delay & $5(\mathrm{~s})$ \\
\hline$w$, Cumulative weight in the recent period & 0.75 \\
\hline
\end{tabular}

We collect real-world traffic data from the Second Ring Road of Changsha, Hunan, China and use them as the leading vehicle's trajectory. Thus, $v_{0}(t), t \in[0,1199] \mathrm{s}$, are calculated based on the trajectory information. The initial displacement of the leader $x_{0}(0)$ is set to 0 , while the followers are located at $x_{n}(0)=x_{n-1}(0)-v_{n}(0) \times \tau-d$ which maintains a minimum safe distance. In the very beginning, the following vehicles' speeds are initiated by $v_{n}(0)=v_{n-1}(0)$.

Figure 2 and Figure 3 show the displacement and speed profiles of the leading vehicle and the first two followers. The red solid line, blue dashed line and green dotted line represent the trajectory/velocity curve of vehicle 0,1 and 2 respectively. We can see that the speed profile of vehicle 1 is much smoother than the leader's, and the vehicle 2's is even smoother than vehicle 1's with cooperative assistance involved. As shown in Table 2, the average speed of vehicles is almost the same while the standard deviation of speed is reduced by $53.5 \%$ to $70.6 \%$.

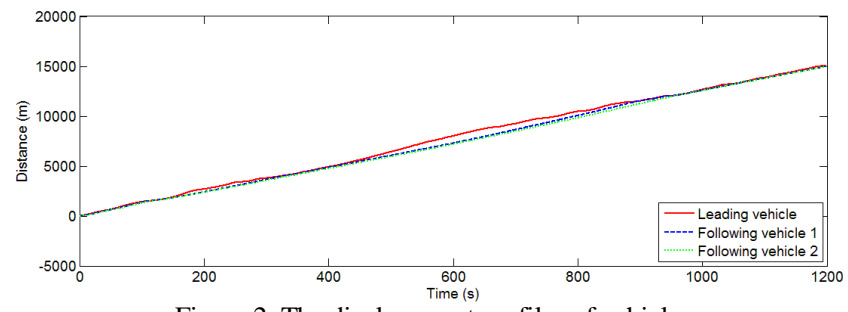

Figure 2. The displacement profiles of vehicles

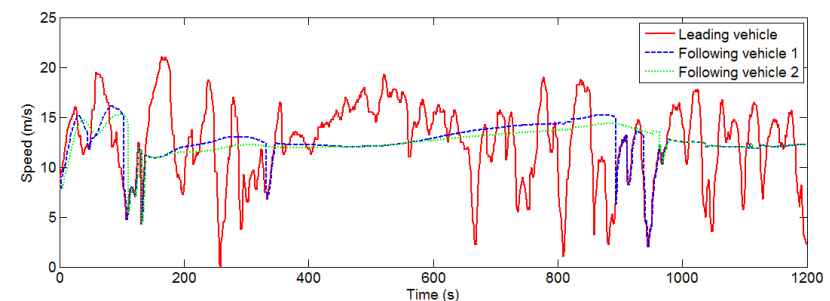

Figure 3. The speed profiles of vehicles 
Table 2. Statistics of speed profiles

\begin{tabular}{|c|r|r|r|r|}
\hline Speed & Min $(\mathbf{m} / \mathbf{s})$ & $\operatorname{Max}(\mathbf{m} / \mathbf{s})$ & $\begin{array}{c}\text { Average } \\
(\mathbf{m} / \mathbf{s})\end{array}$ & $\begin{array}{c}\text { Std. dev. } \\
(\mathbf{m} / \mathbf{s})\end{array}$ \\
\hline The leader & 0.000 & 21.000 & 12.537 & 4.039 \\
\hline Vehicle 1 & 2.000 & 16.125 & 12.515 & 1.879 \\
\hline Vehicle 2 & 4.250 & 15.288 & 12.512 & 1.288 \\
\hline Vehicle 3 & 4.250 & 14.884 & 12.508 & 1.186 \\
\hline
\end{tabular}

Table 3 shows the air emissions and fuel consumption calculated by the VT-Micro software. The linear, quadratic, cubic and quartic coefficients of speed and acceleration are preset based on tests with chassis dynamometer data collected at the Oak Ridge National Laboratory (ORNL). As listed in Table 3, the followers' emissions and consumption are significantly reduced. For example, $\mathrm{CO}_{2}$ emission is reduced by up to $26.9 \%$ and fuel consumption can be saved by $24 \%$ to $28 \%$ with our algorithm implemented. In general, the results of this simulation demonstrate the effectiveness and environmental benefits of our algorithm as expected.

Table 3. Results of pollutant emissions and fuel consumption

\begin{tabular}{|c|c|c|c|c|r|}
\hline Emissions & $\begin{array}{c}\mathbf{C O}_{\mathbf{2}} \\
(\mathbf{g} / \mathbf{k m})\end{array}$ & $\begin{array}{c}\mathbf{C O} \\
(\mathbf{g} / \mathbf{k m})\end{array}$ & $\begin{array}{c}\mathbf{H C} \\
(\mathbf{g} / \mathbf{k m})\end{array}$ & $\begin{array}{c}\text { NOx } \\
(\mathbf{g} / \mathbf{k m})\end{array}$ & $\begin{array}{c}\text { Fuel use } \\
(\mathbf{L} / \mathbf{k m})\end{array}$ \\
\hline The leader & 284.738 & 4.723 & 0.213 & 0.330 & 0.125 \\
\hline Vehicle 1 & 218.502 & 1.442 & 0.091 & 0.104 & 0.095 \\
\hline Vehicle 2 & 209.485 & 1.299 & 0.086 & 0.084 & 0.091 \\
\hline Vehicle 3 & 208.234 & 1.575 & 0.093 & 0.074 & 0.090 \\
\hline
\end{tabular}

\section{CONCLUSION}

In this paper, we introduce Newell's car-following model to describe vehicle movements and FFT algorithm to analyze characteristics in frequency domain. An inter-vehicle communication based algorithm which suggest smoothed speed in stop-and-go traffic is proposed. The algorithm first extracts the period of oscillations, then calculate, adjust and smooth the average speed as the advisory speed with vehicle cooperation involved. In the simulation, we use VT-Micro to compute pollutant emissions and fuel consumption. By comparing the emission data combined with the statistics of speed, we demonstrate the smoothing effect and environmental benefits of our algorithm.

In our study, we focus on a single-lane scenario which is relatively simple. In the real world, multi-lane roads are commonly exist, and the study in traffic models and driver behaviors in multi-lane scenario are necessary and attractive. In addition, communication range and connection loss should be taken into account. Further research are needed to develop more complex algorithms or strategies to make the transportation environmentally friendly.

\section{REFERENCES}

[1] Hennessy, K. B., et al. "Australia and New Zealand: climate change 2007: impacts, adaptation and vulnerability: contribution of Working Group II to the Fourth Assessment Report of the Intergovernmental Panel on Climate Change." (2007).

[2] Alsabaan, Maazen, et al. "Vehicular networks for reduction of fuel consumption and $\mathrm{CO} 2$ emission." Industrial Informatics (INDIN), 2010 8th IEEE International Conference on. IEEE, 2010.
[3] Barth, Matthew, and Kanok Boriboonsomsin. "Real-world carbon dioxide impacts of traffic congestion." Transportation Research Record: Journal of the Transportation Research Board 2058.1 (2008): 163-171.

[4] Smulders, Stef. "Control of freeway traffic flow by variable speed signs." Transportation Research Part B: Methodological 24.2 (1990): 111-132.

[5] Wilkie, Julia K. Using variable speed limit signs to mitigate speed differentials upstream of reduced flow locations. No. SWUTC/97/72840-00003-2. 1997.

[6] Lin, Pei-Wei, Kyeong-Pyo Kang, and Gang-Len Chang. "Exploring the effectiveness of variable speed limit controls on highway workzone operations." Intelligent transportation systems. Vol. 8. No. 3. Taylor \& Francis Group, 2004

[7] Hegyi, Andreas, Bart De Schutter, and Hans Hellendoorn. "Model predictive control for optimal coordination of ramp metering and variable speed limits." Transportation Research Part C: Emerging Technologies 13.3 (2005): 185-209.

[8] Barth, Matthew, and Kanok Boriboonsomsin. "Energy and emissions impacts of a freeway-based dynamic eco-driving system." Transportation Research Part D: Transport and Environment 14.6 (2009): 400-410.

[9] Carsten, Oliver MJ, and F. N. Tate. "Intelligent speed adaptation: accident savings and cost-benefit analysis." Accident Analysis \& Prevention 37.3 (2005): 407-416.

[10] Recker, Will, et al. "Autonet: Inter-vehicle communication and network vehicular traffic." International journal of vehicle information and communication systems 1.3 (2008): 306-319.

[11] Hao Yang, and Wen-Long Jin. "A control theoretic formulation of green driving strategies based on inter-vehicle communications." Transportation Research Part C: Emerging Technologies, Vol. 41 (2014): 48-60.

[12] Newell, Gordon Frank. "A simplified car-following theory: a lower order model." Transportation Research Part B: Methodological 36.3 (2002): 195-205.

[13] Rakha, Hesham, Kyoungho Ahn, and Antonio Trani. "Development of VT-Micro model for estimating hot stabilized light duty vehicle and truck emissions." Transportation Research Part D: Transport and Environment 9.1 (2004): 49-74.

[14] Zielke, Benjamin A., Robert L. Bertini, and Martin Treiber. "Empirical measurement of freeway oscillation characteristics: an international comparison." Transportation Research Record: Journal of the Transportation Research Board 2088.1 (2008): 57-67.

[15] Li, Xiaopeng, Fan Peng, and Yanfeng Ouyang. "Measurement and estimation of traffic oscillation properties." Transportation Research Part B: Methodological 44.1 (2010): 1-14.

[16] Ranjitkar, Prakash, Takashi Nakatsuji, and A. Kawamura. "Carfollowing models: an experiment based benchmarking." Journal of the Eastern Asia Society for Transportation Studies 6 (2005): 15821596.

[17] Ahn, Soyoung, Michael J. Cassidy, and Jorge Laval. "Verification of a simplified car-following theory." Transportation Research Part B: Methodological 38.5 (2004): 431-440.

[18] Chen, Danjue, et al. "A behavioral car-following model that captures traffic oscillations." Transportation research part B: methodological 46.6 (2012): 744-761.

[19] Van Loan, Charles. Computational frameworks for the fast Fourier transform. Vol. 10. Siam, 1992.

Ahn, Kyoungho, et al. "Estimating vehicle fuel consumption and emissions based on instantaneous speed and acceleration levels." Journal of Transportation Engineering 128.2 (2002): 182-190. 